\title{
Measurement of Turbid Body Optical Properties Using Attenuation of Laser Light Intensity Techniques
}

\author{
Senbeto Kena Etana \\ Department of Physics, College of natural and Computational Science, Wollega University, \\ P.O Box 395, Nekemte, Ethiopia
}

\begin{tabular}{|c|c|}
\hline Abstract & Article Information \\
\hline \multirow{3}{*}{$\begin{array}{l}\text { The main objective of this work was to investigate the laser light interaction with highly } \\
\text { diffusing tissue media like milk. In this work collimated transmission, fluence rate } \\
\text { measurements and angular distribution of intensity measurements were made on three } \\
\text { types of milks having different fat contents such as fresh cow milk }(3.90 \%) \text {, skimmed milk } \\
(2.8 \%) \text { and powdered milk and absorption coefficient } \mu_{a} \text {, scattering coefficient } \mu_{s} \text {, reduced } \\
\text { scattering coefficient } \mu_{s}^{\prime} \text {, anisotropic factor } \mathrm{g} \text {, scattering particle diameter } \mathrm{d} \text { and particle size } \\
\text { parameter } \mathrm{X} \text { for different milks were observed. The results of the study revealed that fresh } \\
\text { cow milk showed more fat content when compared to skimmed milk and powdered milk. } \\
\text { Finally, the fluence rate measurement methods may be used to test the quality of milk more } \\
\text { accurately. }\end{array}$} & $\begin{array}{l}\text { History: } \\
\text { ed : } 15-0\end{array}$ \\
\hline & \\
\hline & \\
\hline
\end{tabular}

\section{INTRODUCTION}

Optical properties of scattering media had been extensively studied in order to understand the interaction between laser light and biological media. In recent years, experimental and theoretical studies have been devoted to precise measurements of absorption and scattering coefficients because of their fundamental and practical interest in medicine (Gwamuri et al., 2006; Karin et al., 2014; Ruikang et al., 2013 and Kye et al., 1998).

\section{Light- Tissue Interaction}

When a beam of light is incident on biological tissue, there are various possibilities of light- tissue interactions. These interactions can occur at the air- tissue interface, or inside the tissue, when the rest of the light penetrates the tissue (Stuhlman, 1943; Markolf Niemz, 2007). Light may be reflected at the surface, whilst inside the tissue it undergoes attenuation due to both absorption and scattering. Various light- tissue interactions such as photochemical, photo-thermal, photo-ablative and electrochemical were observed to alterations of the tissue structure or compositions (Glasser,1944). This change of tissue optics alters the rate of energy deposition in the tissue (Jacques, 1993).

Absorption and scattering are reductions in intensity of light beam traversed through the medium having the thickness x. Using the Beer- Lambert law (Hubbell, 1999), one can obtain the intensity $\mathrm{I}(\mathrm{x})$ transmitted through the medium, as

$$
I(x)=I_{0} \exp \left(-\mu_{t} x\right)
$$

Where, $I_{0}$ is the intensity of the incident light, $I$ is the intensity of the transmitted light and $\mu_{\mathrm{t}}$ is total attenuation coefficient, which can be interpreted as the probability that the photon will be attenuated due to absorption and scattering by the medium per unit length. The reciprocal, $1 / \mu_{t}$ is called the attenuation path length and equal to the mean free path of the photon travels between successive attenuation events. This distance required for the intensity of the beam to fall to $1 / \mathrm{e}$ of the initial value (Jenkins and White, 1976).

The scattering coefficient in terms of particle number density $\rho$ and scattering cross section is $\sigma_{c}$

$$
\mu_{s}=\rho \sigma_{c}
$$

The scattering cross section describes the ability of the particle to what extent it scatters light.

The total attenuation coefficient is the sum of absorption and scattering coefficients; i.e.,

$$
\mu_{t}=\mu_{a}+\mu_{s}
$$

The reduced or transport scattering coefficient, is defined as,

$$
\mu_{s}{ }^{\prime}=\mu_{s}(1-g)
$$

Light propagation in highly turbid medium, like biological tissue, can be considered as a process of energy diffusion. Using diffusion approximation method of solving radiation transport equation, one can solve the diffusion coefficient $D$ for homogenous diffusing media (Zaccanti, 1995) is found to be,

$$
D=\frac{1}{3 \mu_{s}(1-g)}
$$

The anisotropy factor, $\mathrm{g}$ is a measurement of the amount of forward direction retained after a single 


\section{Senbeto Kena Etana}

scattering event. The fluence rate as a function of distance between the sample and the detector $r$, is defined as

$$
\phi(r)=\frac{1}{4 \pi D r} \exp \left(-\mu_{e f f} r\right) .
$$

Where $\mu_{\text {eff }}$ the effective attenuation coefficient, which can be obtained from (5) and (6)

$$
\mu_{\text {eff }}=\sqrt{\frac{\mu_{a}}{D}}=\sqrt{3 \mu_{a}\left(\mu_{a}+\mu_{s}^{\prime}\right)}
$$

Rearranging ( $6 \& 7)$, we get,

$$
\ln (\phi(\mathrm{r}) \cdot \mathrm{r})=\ln \left(\frac{1}{4 \pi \mathrm{D}}\right)-\mathrm{r} \mu_{e f f}
$$

Equation (1) can be expressed for total attenuation coefficient $\mu_{t}=c \varepsilon_{t}$ as

$$
I=I_{0} \exp \left(-\mu_{t} L\right)=I_{0} \exp \left(-c \varepsilon_{t} L\right)
$$

Here $\mathrm{c}$ is the concentration of the sample, $\varepsilon_{t}$ is tota extinct coefficient and $L$ is the thickness of the sample. When light from $\mathrm{He}-\mathrm{Ne}$ laser source passes through spherical scattering particle, it does not produce a bright dot as image, but rather a diffuse circular disc known as Airy disc [Jenkins and White, (1976)] and which is given by diffraction equation,

$$
d=\frac{2(1.22)}{\theta} \lambda
$$

The spherical scattering particle size may be computed using the value of $d$. $\theta$ from the above expression represents the angle from the transmission line to where the first minima $(I=0)$ occur. The particle size parameter $X$ can then be calculated as,

$$
X=\frac{d x \pi n_{\text {med }}}{\lambda}
$$

Where $n_{\text {med }}$ is the idex of refraction of the surrounding medium (water). The number density of the particle can be found,

$$
\rho_{s}=\frac{f_{V}}{\frac{4 r^{3}}{3}}
$$

$f_{v}$ is the fraction volume of a sample. The index of refraction of fresh milk, $n_{p}=1.467$ and the surrounding medium (water) $n_{\text {med }}=1.33$. The relative index of refraction,

$$
m=\frac{\mathrm{n}_{\mathrm{p}}}{\mathrm{n}_{\text {med }}}
$$

The scattering cross section, $\sigma_{c}$ for small spherical scattering particle (Jackson,1999), can be ,

$$
\sigma_{C}=\frac{8 \pi}{3} r^{6} k^{4}\left[\frac{m^{2}-1}{m^{2}+1}\right]^{2}
$$

Where $\mathrm{k}$ is a wave number $\left({ }^{k=\frac{2 \pi}{\lambda}}\right)$ and $\mathrm{r}$ is the radius of scattering particle.

\section{MATERIALS AND METHODS \\ Materials}

The materials used for conducting the experiments were a $5 \mathrm{mw} \mathrm{He}-\mathrm{Ne}$ laser source emitting at a wavelength of $632.8 \mathrm{~nm}$, operating in continuous wave $(\mathrm{CW})$ mode,
Sci. Technol. Arts Res. J., Jan-March 2014, 3(1): 53-56

milk was used as diffusing medium and the Indian ink as absorbing medium, sample cells of different size and thickness were used. A lock-in-amplifier, photo- detector, chopper and a filter were used for these experiments.

\section{Collimated Transmission Measurement}

$\mathrm{He}-\mathrm{Ne}$ laser light was directed to transparent scattering cell $10 \mathrm{~mm}$ containing distilled water. The collimated transmitted light that passed through the sample was also passed through the diaphragm (collimator). This was done to avoid the scattering light entering the detector.

\section{Fluence Rate Measurement}

A multi- distance measurement of spatially fluence rate (emittance) inside a highly diffusing medium was carried out. A He-Ne laser source, linearly polarized light emitting at $632.8 \mathrm{~nm}$ with an output power source $5 \mathrm{mw}$ in continuous wave (CW) was used. A chopper was placed orthogonal to the laser beam serving as the beam modulator. The reference signal was obtained from the chopper controller to the lock-in-amplifier. A lens was used for coupling the beam to the optical fiber with the isotropic spherical tips and incident on the diffusion medium through the source or output fiber (Wilson, 1995; Ashley et al., 2011).

\section{Angular Distribution of Intensity Measurement}

A $5 \mathrm{mw}$ He-Ne beam was incident on a sample cell containing $100 \mathrm{ml}$ of distilled water. A $0.1 \%$ by volume of milk sample was dissolved in to water. The intensity of light in arbitrary unit (au) was measured by varying the angle around the sample from the line transmission using the telescope of the spectrometer.

The source and the detection fibers were initially positioned at a distance of $10 \mathrm{~mm}$ held in the diffusing medium (milk) and $30 \mathrm{~mm}$ from the wall of the scattering cell of thickness $6.5 \mathrm{~cm}$ containing $240 \mathrm{ml}$ of distilled water. The source - detector distance $r$ was gradually increased in steps of $5 \mathrm{~mm}$. A $30 \mathrm{ml}$ of milk of was dissolved in to the water. The Indian ink was used as an absorber. The concentration of the ink was gradually increased from 0.000062 to 0.000246 by volume in different steps. For concentration of the added absorber the fluence rate was measured. In this experiment the added absorber (Indian ink) was used to vary the absorption property of the medium (Wilson, 1995; Ashley et al., 2011).

\section{RESULTS AND DISCUSSIONS}

The fat concentration of powdered milk or NIDO (brand name of powder milk) is $28 \% .5 \mathrm{~g}$ of it was dissolved in $100 \mathrm{ml}$ of distilled water, and then the concentration of the fat became $1.33 \%$ i.e.

$$
5 / 100 * 28=1.4 \mathrm{~g}, \frac{1.4}{1.4+100} * 100=1.33
$$

The intensity of transmitted light has been measured using the experimental set up as discussed above and with the help of equation 9, values of were plotted against the concentration of the fresh cow milk. Total attenuation coefficient has been determined from the slope of the graph shown in Figure 1.

The values of extinction coefficient for fresh milk, skimmed milk and powder milk were found to be $\varepsilon_{t}$ $=27.82 \mathrm{~mm}^{-1}, 16.29 \mathrm{~mm}^{-1}$ and $14.57 \mathrm{~mm}^{-1}$, respectively. 


\section{Senbeto Kena Etana}

Using equation (8), values of against $r$ was plotted for each concentration of added absorber. The effective attenuation coefficient, $\mu_{e f f}$, of a medium was evaluated for each added absorber as shown in figure 2 .

From the slope of the figure 2 the effective attenuation coefficient has been determined and from the linear fit of variation of $\mu_{e f f}{ }^{2}$ with $\Delta \mu_{a}$, the absorption coefficient and reduced scattering coefficients were found to be $\Delta \mu_{a}$ $=3.35 \times 10^{-4} \mathrm{~mm}^{-1}$ and $\mu_{s}^{\prime}=0.354 \mathrm{~mm}^{-1}, \mu_{a} \cong 0$. In the result obtained there was no absorption of light by milk at this wavelength (milk is nearly pure scatterer giving the color of it white) as shown in figure 3.

The results shown us $\mu_{a}<<\mu_{s}^{\prime}, \mu_{t} \approx \mu_{s}=C \varepsilon_{s}=(30 \mathrm{ml} /$ $240 \mathrm{ml}) \times 16.29=2.0363 \mathrm{~mm}^{-1}$, using (4) the anisotropy factor $\mathrm{g}=0.826$.

In similar procedure, the measurements were carried out for others milk type. Then the results were summarized as in Table 1.

Table 1 indicates that milk of different fat concentration have different value of optical properties. The reduced scattering coefficient $\mu_{s}^{\prime}$ and anisotropy factor g obtained in this work with that of the literature value, $\mu_{s}^{\prime}=$ $0.59 \mathrm{~mm}^{-1}$ and $\mathrm{g}=0.96$ (Zijp and Bosch, 1998), showed
Sci. Technol. Arts Res. J., Jan-March 2014, 3(1): 53-56

very good agreement. The value $\mathrm{g}$ in the nearest of unity showed us the scattered light is in forward direction.

From figure 4 multi -angular scattering of the particle diameter and scattering particle size parameter were determined for different milk types.

Using the diffraction formula (10), the diameter of the spherical particle was found to be $d=0.936 \mu \mathrm{m}$ and from this by (11) the particle size parameter $X=6.18$ has been determined. Using fractional volume the number density, $\rho_{\mathrm{S}}=0.002325 \mu \mathrm{m}^{-3}$ was obtained.

Mie theory calculation yields the scattering efficiency, which relates the scattering cross section $\sigma_{s}$ to the true geometrical area $\left(A=\pi r^{2}\right)$ as $\sigma_{s}=A Q s$, and from this relation scattering cross section for fresh milk was found to be $\sigma_{s}=1.503 \mu \mathrm{m}^{2}$. In similar procedure, the experiment was carried out for other types of milk, and the result was summarized which have been shown in Table 2.

The above two tables (table 1 and table 2) show that the larger the diameter of the scattering particle, the larger the light will scattered. From the milk type, fresh cow milk has large diameter of scattering particle which is due to high concentration fat content.

Table 1: Summery of Optical properties of different milk.

\begin{tabular}{lccccc}
\hline Phantom Tissue & Concentration C(v/v) & $\boldsymbol{\varepsilon}_{\boldsymbol{s}}\left(\boldsymbol{m} \boldsymbol{m}^{\mathbf{- 1}}\right)$ & $\boldsymbol{\mu}_{\boldsymbol{s}}^{\prime}\left(\mathbf{m m}^{\mathbf{- 1}}\right)$ & $\boldsymbol{\mu}_{\boldsymbol{s}}\left(\boldsymbol{m m}^{\mathbf{- 1}}\right)$ & $\mathbf{g}$ \\
\hline Fresh cow milk & 0.125 & 27.97 & 0.559 & 3.496 & 0.84 \\
Skimmed milk & 0.125 & 16.29 & 0.354 & 2.036 & 0.83 \\
Powder milk & 0.125 & 14.57 & 0.341 & 1.821 & 0.81 \\
\hline
\end{tabular}

Table 2: Summery for values obtained from angular distribution of intensity measurement.

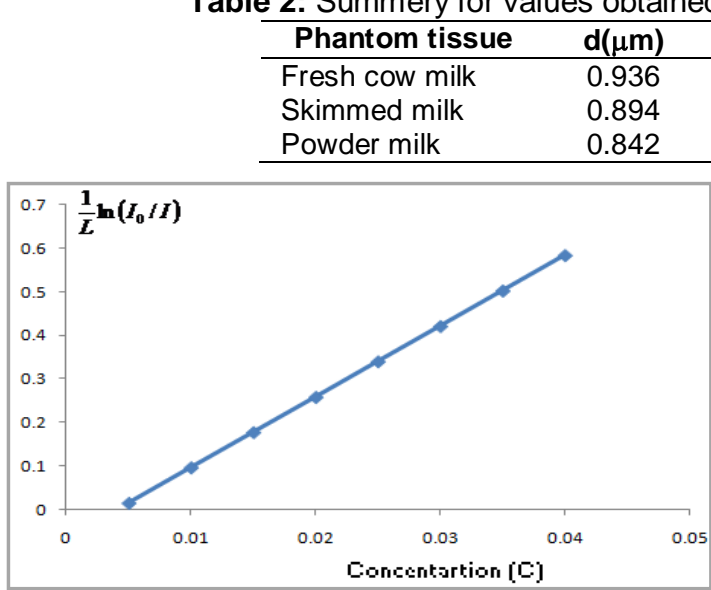

Figure 1: Variation of $\frac{1}{L} \ln \left(I_{0} / I\right)$ with concentration of Fresh cow milk (v/v)

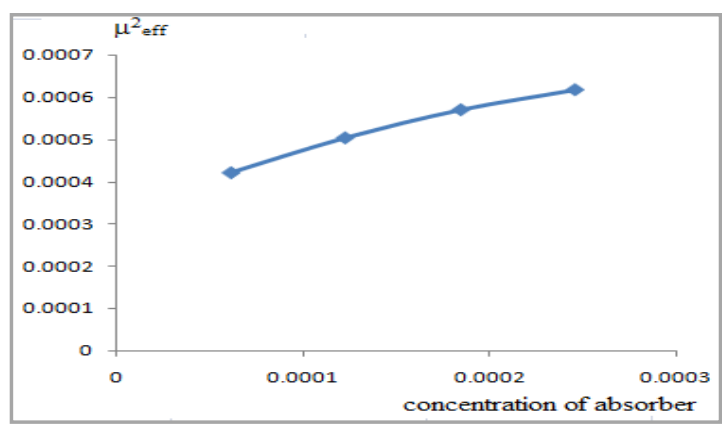

Figure 3: Variation of $\mu_{e f f}{ }^{2}$ with $\Delta \mu_{a}$ for skimmed milk.

\begin{tabular}{cccc}
$\mathbf{A}\left(\mu \mathbf{m}^{2}\right)$ & $\sigma_{\mathrm{s}}\left(\mu \mathbf{m}^{2}\right)$ & $\mathbf{Q}_{\mathrm{s}}$ & $\mathbf{X}$ \\
\hline 0.688 & 1.503 & 2.185 & 6.18 \\
0.632 & 0.883 & 1.398 & 5.91 \\
0.556 & 0.570 & 1.025 & 5.56 \\
\hline
\end{tabular}

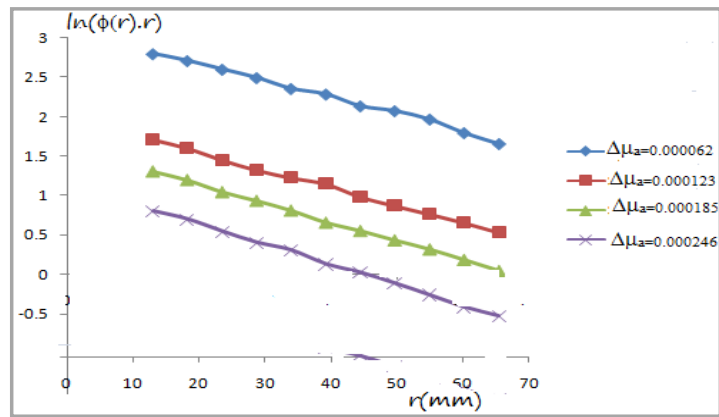

Figure 2: Variation of $\ln (\phi(r) . r)$ with $r$ for skimmed milk for each added absorber

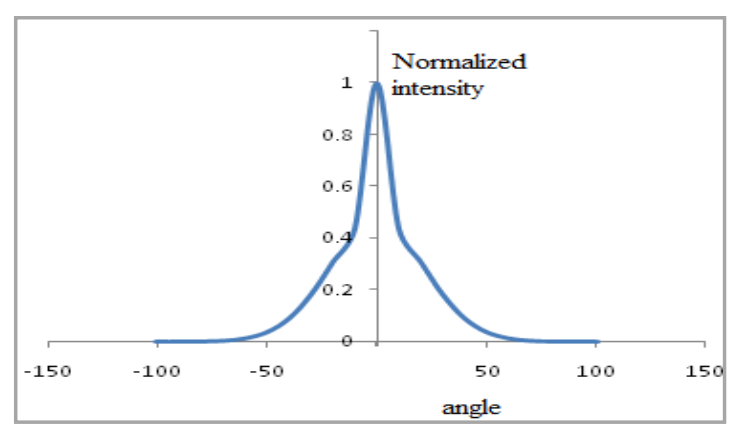

Figure 4: Angular distribution of intensity for fresh cow milk. 


\section{Senbeto Kena Etana}

The results of optical properties of the phantom tissue (milk in this case) were obtained as follows: for fresh cow milk $\left(\mu_{s}^{\prime}=0.559 \mathrm{~mm}^{-1}, \mathrm{~g}=0.84\right)$ which were in good agreement with the reported values (Zijp and Bosch, 1998; Gwamuri et al., 2006, Alexey et al., 2011).

\section{CONCLUSION}

In this work we have been able to investigate fat concentrations with high accuracy light scattering technique, which are in good agreement with reported data. So, these experimental methods can be used to monitor the quality of milk products. This work also suggests that Milk can be used as a phantom tissue in light propagation experiments instead of intra lipid as it is inexpensive, readily available and easy to preserve at room temperature during the experimenting period.

\section{REFERENCES}

Alexey N. Bashkatov., Elina A. Genina. and Valery V. Tuchin (2011). Optical properties of skin, subcutaneous and muscle tissues: A Review, Journal of Innovative Optical Health Sciences 4: 9-38

Ashley J. Welch., Martin J.C., Van Gemert., Willem, M. Star (2011). Definitions and Overview of Tissue Optics in Optical-Thermal Response of Laser-Irradiated Tissue. Springer verlag, US, pp 27-64

Glasser, O. (1944). Medical Physics, The Year Book Publishers Inc., Chicago, Illinois.

Gwamuri, J., Gholap, A.V., Shartir, T.S.M. and Bassuah, P.K. (2006). Investigating Light Propagation in Turbid Media by Evaluating Optical Properties of Phantom Tissues. Proceedings from the $2^{\text {nd }}$ International Conference on Appropriate Technology. Bulawayo, Zimbabwe, July 1215.

Hubbell, J.H. (1999). Review of photon interaction cross section data in Medical and Biological context. Physics in Medicine and Biology 44: R1-22
Sci. Technol. Arts Res. J., Jan-March 2014, 3(1): 53-56

Jackson, J.D. (1999). Classical electrodynamics, $3^{\text {rd }}$ edition, John Wiley and Sons, INC, New York, pp. 459.

Jacques, S.L. (1993). Roll of tissue optics and pulse duration on tissue effects during high power laser irradiation. Applied Optics 32:2447-2454.

Jenkins, F.A. and White, H.E. (1976). Fundamentals of Optics, $4^{\text {th }}$ ed, McGraw Hill, Inc.,New York.

Karin M. Asplund., Kenneth A. Schenkman., Wayne A. Ciesielski., Lorilee, S.L., Arakaki (2014). Photon path depth in tissue phantoms: A comparison of visible and near-infrared(NIR) wavelengths. Proceedings of SPIE 8945; 89450D.

Kye Jin Jeon., Gilwon Yoon., Hyo Soon Eom and Dongho Kim. (1998). Measurement of the Optical Coefficients of Multiple Scattering Media from Time-Resolved Reectance Spectra. Journal of the Korean Physical Society 32: 823827.

Markolf H. Niemz (2007). Laser-Tissue Interactions: Fundamentals and Applications $3^{\text {rd }}$ enlarged ed. Springer, Berlin.

Ruikang K. Wang., Valery V. Tuchin (2013). Optical Coherence Tomography: Light Scattering and Imaging Enhancement Handbook of Coherent-Domain Optical Methods, Springer, Pp.665-742.

Stuhlman, O. (1943). Introduction to biophysics, John Willey and Sons Inc., London.

Wilson, B.C. (1995). Measurement of tissue optics. Plenum press, New York.

Zaccanti, G. (1995). Photon migration through biological tissue, International center for Theoretical Physics, Florence.

Zijp, J.R. and Bosch, J.J. (1998). Optical properties of bovine muscle tissue in vitro; A comparison of methods, IOP publishing Ltd., Netherlands. 